\title{
Causal sets and conservation laws in tests of Lorentz symmetry
}

\author{
David Mattingly \\ University of New Hampshir乎
}

\begin{abstract}
Many of the most important astrophysical tests of Lorentz symmetry also assume that energymomentum of the observed particles is exactly conserved. In the causal set approach to quantum gravity a particular kind of Lorentz symmetry holds but energy-momentum conservation may be violated. We show that incorrectly assuming exact conservation can give rise to a spurious signal of Lorentz symmetry violation for a causal set. However, the size of this spurious signal is much smaller than can be currently detected and hence astrophysical Lorentz symmetry tests as currently performed are safe from causal set induced violations of energy-momentum conservation.
\end{abstract}

\section{INTRODUCTION}

The search for a complete and experimentally verified theory of quantum quantum gravity is one of the most important open questions in physics today. Unfortunately, despite the efforts of numerous eminent physicists, we do not yet have a theoretically complete model for quantum gravity. Since the natural energy scale of quantum gravity is the Planck scale $\left(M_{P}=10^{19} \mathrm{GeV}\right)$ it is also extremely difficult to perform direct experiments that support one candidate model for quantum gravity over another. Fortunately, various ideas about quantum gravity have suggested that the defining symmetry of special relativity, Lorentz symmetry, is not an exact symmetry even at low energies. In string theory this can occur perhaps by tensor VEV's [1] or noncritical strings [2, 3] while in loop quantum gravity the ultimate fate of Lorentz symmetry and how it's implemented is an open question (c.f. [4, 5, 6, 7] ). Emergent spacetime models, for example analog spacetime [8], contain Lorentz symmetry violation (LV) at high energies. Canonical noncommutative field theories also contain Lorentz violation [9, 10, 11] although they are invariant under the twisted Poincare group [12]. Note that there are also noncommutative models where Lorentz invariance is deformed rather than explicitly violated (see [13, 14] and references therein).

Of course any violation of Lorentz invariance must be very, very small and therefore for any model with LV there is a severe hierarchy problem. For example, in an effective field theory context, mass dimension three operators that generate Lorentz symmetry violation must be less than $10^{-32} \mathrm{GeV}$ while the dimension four operators are constrained at the level of $10^{-28}$ [15]. These extreme bounds mean that any Lorentz violating theory must answer the question of why we have such good approximate low energy Lorentz invariance. One could fine tune operators, but this is unnatural. In an attempt to avoid this, many authors have looked at higher dimension operators where the magnitude of the operator is suppressed by $M_{P}$ and so is "hidden". However, standard

*Electronic address: davidmmattingly@comcast.net effective field theory arguments require that the higher dimension operators dimensionally transmute into lower dimension ones [16]. This means that dimension five and six operators are constrained at the same order as the dimension three and four operators and so LV alone is extremely unlikely. It is possible to naturally suppress the renormalizable operators by introducing new physics at scales just above that currently accessible by particle detectors. For example, imposing supersymmetry 17] as well as Lorentz violation stabilizes the generation of lower dimension operators at the SUSY breaking scale. If the SUSY breaking scale is given by $m_{S U S Y}$, and the original higher dimension operators are suppressed by $M_{P}$, then SUSY will naturally suppress the dimension three and four operators to the level of $m_{S U S Y}^{2} / M_{P}$ and $m_{S U S Y}^{2} / M_{P}^{2}$ respectively. For $m_{S U S Y}<100 \mathrm{TeV}$, this gives a suppression to a level of approximately $10^{-9} \mathrm{GeV}$ and $10^{-28}$. The dimension three operators are still in conflict with observational bounds, but these can be eliminated by further imposing CPT invariance. This would make a model with low energy SUSY and CPT invariance and LV sourced by Planck scale quantum gravity viable, but requires significant new low energy physics as well (in this case SUSY and the assumption of CPT).

There is, of course, nothing a priori wrong with introducing new low energy physics and SUSY certainly has a number of other compelling features unrelated to LV. If we restrict ourselves just to the question of LV, however, then Occam's razor suggests that adding two new physical ideas is disfavored. Hence, instead of either fine tuning or imposing new low energy physics in addition to $\mathrm{LV}$, it would be better perhaps to have a quantum gravity theory that respects Lorentz invariance exactly. The discrete model we will discuss for the rest of this paper, causal sets, is singled out among discrete models as it is constructed to be Lorentz invariant by definition. Recently, Sorkin, Bombelli, and Henson [18] proved that a causal set is Lorentz invariant for an abstract operator that represents a measurement of a preferred frame for a section of the causal set (the proof is that such operators cannot exist). In this work we argue that this operator $D$ does not quite reflect the way that we currently analyze many real Lorentz violating experiments and that such experiments (in particular astrophysical tests) may theoretically show "spurious" Lorentz violating effects if the 
underlying spacetime is a causal set. However, we also show that any effect is much smaller than our current experimental sensitivities and can be safely ignored.

The essence of our argument is that swerves, a hypothetical effect on particle propagation in causal set theory [19], can mimic a signal of LV in time of flight or threshold astrophysics experiments where the expected flux of incoming high energy particles is very low. This may seem strange, as causal sets are supposed to not give any LV signal. However, swerves violate energymomentum conservation, which we usually assume holds exactly in the vacuum when we analyze LV experiments. This mismatch between theory and assumption leads to a spurious LV signal. Violations of energy-momentum conservation are tightly constrained from cosmology and the constraints are strong enough that this effect is irrelevant at current sensitivities in LV experiments but may not be in the future. Furthermore, other ideas about quantum gravity have raised the spectre of a small violation of energy-momentum conservation for particles traveling in the vacuum(c.f [20, 21, 22]), so exploring how this changes LV experiments is required if we are to analyze the experiments properly.

An added benefit to this study is that, for a population of particles, the violation of energy-momentum conservation predicted from causal sets satisfies a low energy diffusion equation in energy-momentum space. This equation is unique, and therefore our result implies that we can rather generically neglect the effects of any stochastic, Lorentz invariant violation of energy-momentum conservation in Lorentz symmetry tests, whether or not we believe in causal sets. Hence while causal sets are the motivation, the results apply more broadly (although causal sets are the only model at this point that hypothesizes such an effect). To put it differently, assuming energymomentum conservation is not a priori warranted when searching for quantum gravity induced LV. However, the constraints on Lorentz invariant conservation violation are so tight that we can safely ignore it at our current level of sensitivity in LV tests, independent of whatever the fundamental theory turns out to be.

Throughout the following discussion we choose metric signature -2 and units such that $\hbar=c=1$.

\section{CAUSAL SETS AND THE POINCARE GROUP}

Before we can discuss the fate of the Poincare group and conservation laws in the context of causal sets, we need to know what exactly a causal set is. At its basic level, a causal set is a partially ordered set (a poset), consisting of 'points' $x, y, \ldots$ and relations $x \prec y$ which encode causal ordering, i.e. $x \prec y$ implies $x$ is to the past of $y$. The ordering obeys two other rules, $x \prec y$, $y \prec z \Rightarrow x \prec z$ and $x \nprec x$. The first is simple transitivity such that if $x$ is in the past of $y$ and $y$ is in the past of $z, x$ had better also be in the past of $z$. The other condition forbids closed causal curves. Causal sets are usually considered to be finite, so for any ordered pair $\{x, y\}$ with $x \prec y$, there are a finite number of points $z$ that satisfy $x \prec z$ and $z \prec y$. While it is certainly not necessary for a spacetime to be present for a causal set to be defined, a useful picture is to think of a causal set as a random lattice "sprinkled" in a Lorentzian spacetime where there is an average of one point per every volume $V_{0}$ (which will be assumed here to be a Planck volume $\left.L_{P l}^{4}\right)$. The causal set sprinkling therefore approximates $M$. For more information on causal sets, see [23] and references therein.

It has been argued that an individual causal set is Lorentz invariant [18], even locally, in a particular sense: there is no experiment that can assign an intrinsic violation of Lorentz invariance to a causal set at any point. Here "intrinsic violation" means a frame that depends only on the sprinkling. We now briefly outline the proof in [18], restricting ourselves to the simplest type of LV a preferred frame. The question is then, can a causal set define such a frame? Certainly a regular discrete structure for spacetime does, but a causal set is a random structure where the points are distributed in spacetime from a Lorentz invariant probability distribution. A preferred frame is specified by the existence, in vacuum, of a unit future pointing timelike vector field $u^{a}$ everywhere on the spacetime manifold $M$. Consider now a subset $\Omega$ of a causal set sprinkling that approximates $M$. An experiment that assigns a LV preferred frame can be represented as an operator $D$ that maps $\Omega$ to the unit hyperboloid of future timelike vectors $H$, i.e. $u^{a}=D \circ \Omega$. $u^{a}$ transforms as an ordinary four vector under a Lorentz transform $\Lambda . D$ must not posses any intrinsic preferred direction (to ensure that any LV comes from $\Omega$ itself) and therefore $D$ must commute with $\Lambda$ so that $D \Lambda \Omega=\Lambda u^{a}$. The proof is then that no such operator $D$ can exist and there is no experiment that can assign a preferred frame to a local patch $\Omega$ of a causal set. The implication is that no LV experiment can ever assign a preferred frame to a causal set intrinsically.

This result, on its face, is incompatible with another hypothesized effect of causal sets, a violation of translation invariance due to the so-called "swerve" effect [19]. The swerve effect, which we discuss in more detail in the next section, manifests itself at low energies via a Lorentz invariant diffusion equation in momentum space. An initial collection of particles with some momentum distribution $\rho(p)$ therefore evolves into a different state over (proper) time, which obviously violates translation invariance. The immediate question is then, how does this not directly yield LV? After all, translation violation allows us to immediately define a frame by taking, for example, the gradient of the evolving quantity as the operator $D$. The answer is that the frame so defined is not intrinsic to the causal set, but also involves the initial distribution $\rho(p)$. In particular, if $\rho(p)$ is a Lorentz invariant function (which is unrealistic physically but useful for this discussion) it is preserved by the diffusion equa- 
tion. Hence there is neither a violation of translation or Lorentz invariance in this case. If, instead, $\rho(p)$ is not Lorentz invariant then there can be translation invariance violation, but both it and the corresponding $\mathrm{LV}$ are functions of the causal set and the initial LV distribution. There is a "signal" of LV, but it is not the intrinsic LV which is forbidden by the proof outlined above because our operator $D$ contains a preferred direction, which violates one of the assumptions in the proof.

The difference between these two types of LV signal a real signal intrinsic to the underlying spacetime vs. a spurious signal due to the combination of the preferred frame of an experiment with another property of spacetime (in this case swerves) is a possibility which has not arisen before in analysis of LV experiments. Since all of our observations and experiments that search for LV are not LI in and of themselves (as they are local and happen in a particular frame) we must consider it. Hence the question of LV in causal sets for a practical experiment is not quite as straightforward as presented in [18]. The particular example we examine in this paper is how the assumption of translation invariance, which is customary in LV experiments, can generate a spurious LV signal from a causal set.

Translation invariance is a bad assumption due to the swerve effect, as we a) single out a preferred Killing vector which is not an intrinsic property of the causal set itself and b) neglect momentum space diffusion due to swerves. The most common astrophysical tests of LV are time of flight tests, where we compare the arrival time of two high energy particles, and anomalous scattering/decay phenomena, where we look for a modification to the scattering amplitude caused by LV dispersion relations for the in/out free particle states. As will become clear below, when analyzing these phenomena we must assume something about translation invariance and conservation of energy and momentum in order to put constraints on any possible LV. Usually, translation invariance has been assumed to hold exactly in flat space, as this is most consistent with a straightforward field theoretical approach if the background LV tensor fields are constant. ${ }^{1}$ With causal sets, this assumption isn't right and we must verify that a LV signal is really due to LV and not the swerve effect.

\footnotetext{
1 There are two major exceptions to this, the doubly special relativity program (see 13] for an introduction) which deforms both the translational and Lorentz subgroups of the Poincare group, and spacetime foam ideas which couple LV to a fluctuating dispersion term 24, 25]. These approaches, however, have a significant a priori modification of both Lorentz invariance and translation invariance which makes them distinct from the causal set approach.
}

\section{A. Swerves}

Consider the intuitive picture for swerves in [19], that of a classical particle propagating on a random spacetime lattice with mass $m$ and velocity $v$. The particle is constrained to move from point to point, which poses a problem as generically there is no probability of a lattice point lying on the future worldline of the particle. The particle must therefore "swerve" slightly so that it remains on the lattice and slightly change its velocity. A change in velocity is equivalent to the particle moving to a different point on its mass shell, which is assumed to be unchanged since causal sets are Lorentz invariant. The net result of swerving is that a collection of particles initially with an energy-momentum distribution $\rho(p)$ will diffuse in momentum space along their mass shell according to the unique Lorentz invariant diffusion equation [19, 26],

$$
\frac{\partial \rho}{\partial \tau}=k \nabla_{P}^{2} \rho-m^{-1} p^{\mu} \partial_{\mu} \rho .
$$

Here $k$ is the diffusion constant, $\nabla_{P}^{2}$ is the Laplacian in momentum space on the mass shell of the particle, $\tau$ is the proper time, $\partial_{\mu}$ is an ordinary spacetime derivative, and $m$ is the mass. While the underlying classical picture is almost certainly incorrect, (11) is the unique Lorentz invariant diffusion equation. Therefore if there is any type of stochastic violation of Poincare invariance due to a random discrete structure underlying spacetime a la causal sets, at lowest order it should be described by (1). There is a very tight limit on $k$ for neutrinos from cosmology [27], $k<10^{-61} \mathrm{GeV}^{3}$, which comes from limits on the amount relic neutrinos can contribute to hot dark matter (and hence how much energy they can gain from swerves). While theoretically each particle species could have a different $k$, it would be rather unnatural if they were too far apart, especially as the source of the diffusion is supposed the same discrete spacetime structure. Therefore we shall take $k<10^{-61} \mathrm{GeV}^{3}$ to be our rough constraint for all particles. As it turns out, any value of $k$ even close to $10^{-61} \mathrm{GeV}^{3}$ makes energy-momentum conservation violation irrelevant for LV searches, so this is a perfectly safe assumption.

Energy is bounded below and so a particle initially at rest will increase its kinetic energy due to swerves. Since $k$ is so low, we can make a simplifying assumption for any collection of particles that are not of cosmological age. In the initial rest frame of the particle the swerves can first be treated in the non-relativistic limit for a certain period of time that depends on $k$. This is obvious as over time energy is being added to the particle via swerves, but as long as the total energy is less than the rest mass the appropriate limit is the non-relativistic one. In the non-relativistic limit, (1) simplifies to

$$
\frac{\partial \rho}{\partial t}=k \nabla_{P}^{2} \rho
$$

where $t$ is now the coordinate time and $\nabla_{P}^{2}$ is the stan- 
dard Laplacian operator on momentum space $\mathbb{R}^{3}$. The solution for a collection of particles all initially at rest has been derived in [27] and is given by

$$
\rho(p)=(4 \pi k t)^{-3 / 2} e^{-\frac{p^{2}}{4 k t}}
$$

which is the thermal distribution for a non-relativistic gas at temperature $T=2 \mathrm{kt} / \mathrm{m}$.

We can now ask how long a collection of initially cold particles must exist for the non-relativistic approximation to break down. This happens when the temperature is roughly equal to the mass, which gives $t \approx m^{2} /(2 k)$. For a neutrino of mass $10^{-1} \mathrm{eV}$, the approximation breaks down after $10^{17}$ seconds, while for electrons and protons the approximation is always good as the breakdown time is far longer than the age of the universe. After a population becomes relativistic it will still gain energy but not as quickly since the proper time is shorter. Therefore we know that the energy gained (per neutrino) by a population of initially cold neutrinos over the lifetime of the universe (also approximately $10^{17}$ seconds) is no more than about the rest mass of the neutrino and the energy gain for other species is far less. This neglects the effects of cooling due to expansion, etc. which are dealt with in [27], however we can ignore these secondary effects for our purposes. Now consider a population of particles with a high gamma factor $\gamma$ that have traveled to earth from a source at one Gpc. The lifetime of the particle in our frame $O$ is $10^{9}$ years $=10^{16}$ seconds. However, the time in the (initial) rest frame $O^{\prime}$ of the particle is much shorter, $10^{16} / \gamma$ seconds. Since any particle can gain no more than $m_{\nu}$ in energy over $10^{17}$ seconds and the propagation time in $O^{\prime}$ is much shorter, very little energy is gained in $O^{\prime}$ due to swerves. Hence in $O^{\prime}$ the particles are all still very non-relativistic once we include the swerve effect as long as $\gamma \gg 1$ and the non-relativistic approximation is valid for their entire lifetime.

Of particular interest is the average deviation from the initial energy $E_{i}$ of a particle. If we define $\Delta E / E_{i}=$ $\left|\left(E_{f}-E_{i}\right)\right| / E_{i}$, where $E_{f}$ is the energy at time of measurement, then from the discussion above we know that $\Delta E / E_{i}$ is at least less than $\gamma^{-1}$ (on average) for any species. We can see this more explicitly by noting from above that for a neutrino with a lifetime of the age of the universe at rest in our frame, the total energy gain is at best the mass of the neutrino. If instead the neutrino is boosted with respect to our frame, the proper time is reduced by a factor of $\gamma^{-1}$ and so the total energy gain is reduced also by $\gamma^{-1}$ from its initial energy $E_{i}=\gamma m$. Therefore for neutrinos the ratio $\Delta E / E_{i} \leq \gamma^{-1}$. For $\mathrm{TeV}$ and above astrophysical neutrinos, which we are primarily interested in, $\Delta E / E_{i} \leq \gamma^{-1}=10^{-13}$ at worst. The swerve rate is slower for other species, so this bound holds for them as well. This gain is very small, however LV tests can be sensitive to fractional changes in energy of order $10^{-28}$ so it is not a priori obvious that swerves are irrelevant. We now turn to how this diffusion affects these LV tests.

\section{SWERVES AND ASTROPHYSICAL TESTS OF LV}

\section{A. Time of flight}

Time of flight tests are perhaps the simplest type of LV experiment. In these experiments one looks for delays in the arrival time of high energy particles from distant astrophysical events. A time of flight experiment compares the arrival time of at least two high energy particles and the time delay between arrivals can be caused by three distinct effects. The first is source effects - the particles are not produced at the same time or location in the astrophysical event. The source delay can be quite long, in the case of neutrinos from gamma ray bursts the delay time of a neutrino associated with the burst can be days. The second type of delay is detector response. These delays are usually small and known and we will not consider them further. Finally, an unexplained time delay is usually considered evidence that the speed of propagation of the high energy particles is different than the speed of light. This is the "interesting" signal of a violation of Lorentz invariance.

\section{Time of flight in field theory}

It will be useful to discuss in detail how these experiments work in a very concrete and established framework first, before we consider the causal set scenario, so let us analyze time of flight in a field theory context first. LV occurs when fields are coupled in vacuum to a non-zero tensor field other than the metric. If there exists a preferred frame in nature, the fields couple to a unit timelike vector $u^{a}$ which describes the preferred frame. There are many ways a field could couple in both the matter and gravitational sectors [28, 29, 30, 31], for a review of both the renormalizable and non-renormalizable operators see [32]. The free field mass dimension five couplings and below are already tightly constrained, so we consider here an unconstrained operator - that of a dimension six CPT even operator. While terms like this may be able to be tested in ultrahigh energy neutrino observatories in the future and hence are intrinsically relevant, we choose this term for another reason: if the swerve effect is irrelevant for tests of this term it is certainly irrelevant for any LV time of flight test we could conceivably perform in the near future. The dimension six CPT even LV modification to the kinetic term for a fermion is

$\mathcal{L}_{f}=\bar{\psi}(i \not \partial-m) \psi-\frac{i}{E_{P l}^{2}} \bar{\psi}(u \cdot \partial)^{3}(u \cdot \gamma)\left(\alpha_{L} P_{L}+\alpha_{R} P_{R}\right) \psi$

where $P_{R, L}$ are the usual right and left chiral projection operators and $\alpha_{R, L}$ are coefficients. Usually $\alpha_{R, L}$ are assumed to be $\mathrm{O}(1)$. We choose the operator to be suppressed by the Planck scale $E_{P l}$ as this would be the natural scale if the term was generated by some theory 
of quantum gravity at $E_{P l}$.

The Hamiltonian corresponding to (4) commutes with the helicity operator, hence the eigenspinors of the modified Dirac equation will also be helicity eigenspinors. We now solve the free field equations for the positive frequency eigenspinor $\psi$. Assume the eigenspinor is of the form $\psi_{s} e^{-i p \cdot x}$ where $\psi_{s}$ is a constant four spinor and $s= \pm 1$ denotes positive and negative helicity. Then the Dirac equation becomes the matrix equation

$$
\left(\begin{array}{cc}
-m & E-s p-\alpha_{R}^{(6)} \frac{E^{3}}{E_{P l}^{2}} \\
E+s p-\alpha_{L}^{(6)} \frac{E^{3}}{E_{P l}^{2}} & -m
\end{array}\right) \psi_{s}=0
$$

The dispersion relation, given by the determinant of (5), is

$$
\begin{array}{r}
E^{2}-\left(\alpha_{R}^{(6)} E^{3}\right)(E+s p) \\
-\left(\alpha_{L}^{(6)} E^{3}\right)(E-s p)=p^{2}+m^{2}
\end{array}
$$

where we have dropped terms quadratic in $\alpha_{R, L}^{(6)}$ as they are small relative to the first order corrections for those terms.

At $E \gg m$, as appropriate for high energy astrophysical particles, the helicity states are almost chiral, with mixing due solely to the particle mass. Note also that at energies $E>>m$, we can replace $E$ by $p$ at lowest order, which yields the approximate dispersion relation

$$
E^{2}=p^{2}+m^{2}+2 \alpha_{R, L} \frac{p^{4}}{E_{P l}^{2}} .
$$

Positive coefficients correspond to superluminal propagation, i.e. $v=\partial E / \partial p>1$, while negative coefficients give subluminal propagation. In either case, the speed of astrophysical particles does not asymptote to $c$ as the energy increases.

The group velocity $\partial E / \partial p$ is

$$
v=1-\frac{m^{2}}{2 p^{2}}+3 \alpha_{R, L} \frac{p^{2}}{E_{P l}^{2}}=1+\Delta v .
$$

For a source at distance $d$ from earth the arrival time $t_{a}$ of a high energy particle is $t_{a}=d / v$. The difference $\Delta t_{L V}$ between a light pulse emitted from the source at the same time as the particle is

$$
\begin{aligned}
\Delta t_{L V}=t_{\text {light }}-t_{a} & =d-\frac{d}{v}=d\left(\frac{v-1}{v}\right) \approx d \Delta v \\
& =d\left(-\frac{m^{2}}{2 E^{2}}+3 \alpha_{R, L} \frac{E^{2}}{E_{P l}^{2}}\right)
\end{aligned}
$$

where we have replaced $p$ by $E$ in the high energy limit.

The $\alpha_{R, L}$ term in (9) grows with energy. In order to establish the best possible constraints we therefore need to look at the highest energy particles. The best chance we have in a time of flight experiment to see LV at this order is in the comparison of the arrival time of high energy neutrinos produced by GRB's with the prompt emission arrival. The flux at these energies is very low which has both positive and negative ramifications. On the negative side we require large detectors like ICECUBE to see any appreciable flux of ultra high energy GRB neutrinos. However the background flux is also very low. Recently, it has been proposed by Jacob and Piran 33. that since the background is so low even the detection of a single neutrino event days or weeks after a GRB can be associated with the GRB and used to bound $\alpha_{R, L}$ in a time of flight experiment. While this approach has other problems, primarily long source delays [34] due to the GRB fireball mechanics, it raises an interesting question with regards to causal sets - what happens in the swerve picture when there are very, very low statistics?

\section{Time of flight with swerves}

In time of flight experiments with large fluxes, swerves aren't a problem. For a strong multiparticle signal the average arrival time is still that as predicted by special relativity. However, for a single particle signal with measured energy $E$ there is no concept of averaging and the arrival time will not be that predicted by special relativity. The reason is simple - during propagation the particle's energy is not $E$ and the particle's velocity is not $v=1-m^{2} /\left(2 E^{2}\right)$. Therefore to conclusively ascribe a time delay to a LV dispersion as in (9) we need to make sure the same delay cannot be due to swerves.

We can overestimate the time of flight delay for a typical particle compared to special relativity by considering a particle propagating with energy $E_{f}+\Delta E$, where $E_{f}$ is the measured (final) energy and $\Delta E$ is the average deviation for a single particle introduced previously. It is an overestimate since we apply the energy difference over the entire propagation of the particle. The dispersion relation is simply the relativistic dispersion relation, so $\Delta t_{S}$ is

$$
\Delta t_{S}=d\left(-\frac{m^{2}}{2\left(E_{f}+\Delta E\right)^{2}}\right)=d\left(-\frac{m^{2}}{2 E_{f}^{2}\left(1+\Delta E / E_{f}\right)^{2}}\right)
$$

$\Delta E / E_{f}<\gamma^{-1} \ll 1$ and we therefore have

$$
\Delta t_{S}=d\left(-\frac{m^{2}}{2 E_{f}^{2}}\left(1-2 \frac{\Delta E}{E_{f}}\right)\right) .
$$

Comparing (11) with (9) we see that an experiment sensitive to LV at our chosen order is also sensitive to swerves if

$$
3 \alpha_{R, L} \frac{E_{f}^{2}}{E_{P l}^{2}} \approx \frac{m^{2}}{E_{f}^{2}} \frac{\Delta E}{E_{f}} .
$$

Rewriting this equation for $\Delta E / E_{f}$ we have

$$
\frac{\Delta E}{E_{f}}=3 \alpha_{R, L} \frac{E_{f}^{4}}{m^{2} E_{P l}^{2}} .
$$


There is no intrinsic size to the LV term in (13) and depending on how accurate a LV time of flight measurement is, it could theoretically also probe swerves. However, the duration of a long GRB can be of order 1000 seconds and it is unclear when in the burst emission the neutrinos will occur. Therefore there is an intrinsic unknown source delay of at least 1000 seconds $^{2}$. Any significant time delay must be greater than this value and hence there is a lower limit on a meaningful $\Delta t$ (swerve or LV induced). For a GRB with this value of $\Delta t$ and a distance of $1 \mathrm{Gpc}, \Delta t / d \approx 10^{-14}$ seconds. With (9) this establishes a lower limit that the LV dispersion term of

$$
\left|\alpha_{R, L} \frac{E_{f}^{2}}{E_{P l}^{2}}\right| \geq 10^{-14}
$$

which must be satisfied if we are to see anything meaningful in a time of flight GRB experiment. If we are conservative and take our high energy neutrinos to be above $1 \mathrm{TeV}$ (actual proposed energies are much higher) and a neutrino mass of approximately $0.1 \mathrm{eV}$, the gamma factor is $10^{13}$. From (13),$\Delta E / E_{f}$ from swerves must then be greater than $10^{12}$, which is 25 orders of magnitude larger than the upper limit for swerving neutrinos. Hence swerves are completely and totally irrelevant. This result can easily be generalized to other forms of LV dispersion and in none are astrophysical time of flight experiments sensitive to swerves by many orders of magnitude.

\section{B. Anomalous particle interactions}

Anomalous particle interactions are much more sensitive tests of LV than time of flight tests and also of course require assumptions about energy-momentum conservation. Therefore they will also be sensitive to the swerve effect at some level. There are two types of anomalous interactions. The first type is when the interaction occurs only in the LV model. The second type is when the interaction begins to occur at a certain energy and this energy is different for Lorentz invariant versus LV models. We deal with each type of interaction separately as the effect of swerves is different. ${ }^{3}$

\footnotetext{
2 The actual value is of course dependent on the exact mechanics of the GRB and can be shorter. We use this value for illustrative purposes as an order of magnitude estimate.

3 We are not considering particle creation due to the time dependence of the spectrum of an initial flux of particles due to swerves or how to correctly define initial/final states in a model without asymptotic translation invariance. These questions must also be answered in regards to the swerve effect but are outside the scope of this paper.
}

\section{New particle interactions}

Consider a proton with dispersion relation like that given in (77). If $\alpha_{R, L}>0$ then at high energies protons become unstable and emit photons in what is known as the "vacuum Cerenkov effect". They rapidly lose energy via this process and hence the existence of ultra high energy cosmic ray protons (for which this process cannot be occurring) limits how positive $\alpha_{R, L}$ can be. This is an example of a test that uses a particle interaction completely absent in usual Lorentz invariant physics to limit possible LV dispersion relations. The key to these tests is the energy-momentum conservation equations, which tell us how much parameter space is available for the reaction - zero in the Lorentz invariant case and nonzero with LV. Naively then, it seems reasonable that a fluctuating energy-momentum of the initial and/or final states may allow for new reactions to also occur. We now show that in the case of causal sets, this is not true. If we consider just a swerve induced change in the energymomentum of the initial and final states then if a reaction does not occur in straightforward special relativity it does not occur in causal sets. Note that in the following discussion we have implicitly assumed that the swerve effect for massless particles yields diffusion in a null cone in energy-momentum space (the $m \rightarrow 0$ limit of a mass shell).

The idea is very simple. Let us consider a generic multi-particle reaction $i_{1}+i_{2}+\ldots \rightarrow f_{1}+f_{2}+\ldots$ where $i_{a}$ are the incoming particles and $f_{b}$ are the outgoing particles. If the reaction does not occur in Lorentz invariant physics it means that there is no solution to the conservation equation

$$
p_{1}^{\mu}+p_{2}^{\mu}+\ldots=q_{1}^{\mu}+q_{2}^{\mu}+\ldots
$$

where $p_{a}^{\mu}$ (the incoming 4-momenta) and $q_{b}^{\mu}$ (the outgoing 4-momenta) are subject to the on-shell constraints $p_{a}^{2}=$ $m_{a}^{2}, q_{b}^{2}=m_{b}^{2}$. In a LV theory the on-shell constraints change, in causal sets they do not. In a causal set, the incoming and outgoing momenta are, however, modified by a fluctuation term $\Delta p_{a}, \Delta q_{b}$ as the particle will swerve during the course of the interaction. We therefore rewrite the conservation equation in the causal set approach as

$$
p_{1}^{\mu}+\Delta p_{1}^{\mu}+p_{2}^{\mu}+\Delta p_{2}^{\mu}+\ldots=q_{1}^{\mu}+\Delta q_{1}^{\mu}+q_{2}^{\mu}+\Delta q_{2}^{\mu} \ldots
$$

subject to the on-shell constraints $\left(p_{a}+\Delta p_{a}\right)^{2}=$ $m_{a}^{2},\left(q_{b}+\Delta q_{b}\right)^{2}=m_{b}^{2}$ since the fluctuations must keep all particles on-shell. This though is just a relabeling of momenta and doesn't change any physics - we can define new momenta $\bar{p}_{a}^{\mu}=p_{a}^{\mu}+\Delta p_{a}^{\mu}$ etc. and the conservation and constraint equations take the exact same form as before. Therefore there is still no solution and the reaction doesn't happen with swerves either.

There is a caveat to the above argument. In keeping with other LV threshold tests, where one looks at only initial and final states, we have not considered the effect of swerves in the interaction region. This is more 
dangerous for causal sets, as field theory on causal sets is not well developed enough to know what the swerve effect might do to virtual states that are not necessarily on-shell. Hence this conclusion can not be considered absolutely concrete until we understand more of quantum field theory on a causal set. However, since no LV reactions have been seen to date it is likely that causal set QFT does respect LI to a very good approximation even when quantum effects are taken into account.

\section{Modification of existing energy thresholds}

The situation is different for reactions where there is a Lorentz invariant solution. Here, the swerve effect can change the energy the reaction occurs at, although the shift is tiny. Let us take a specific reaction, pion production by proton-photon scattering, $p+\gamma \rightarrow p+\pi^{0}$. This reaction is important in LV tests as the high energy cosmic ray spectrum should exhibit a cutoff (the Greisen-Zapsetin-Kuzmin cutoff) around $5 \times 10^{19} \mathrm{eV}$ due to the scattering of cosmic ray protons off the cosmic microwave background. LV tests can shift this cutoff, and the recent confirmation of the GZK cutoff by HiRes 35] and the Pierre Auger observatory [36] constrains some LV models. Again, we have a limited number of events (although with Auger the statistics are getting rapidly better) and so one might wonder if the random nature of swerves can cause a spurious signal. Theoretically this is true, but the effect of swerves on GZK protons is negligible, even if we wildly overestimate the length of time swerves have to act. A GZK proton has a gamma factor of near $10^{10}$, which means that its maximum proper lifetime is $10^{17} / \gamma=10^{7}$ seconds if it was generated very early in the universe. The maximum amount of energy the proton can gain in its initial rest frame, using our constraint for $k$, is $10^{-30} \mathrm{GeV}$, which in turn implies that the shift in energy possible for a GZK proton in our frame is $10^{-20} \mathrm{GeV}$. The GZK cutoff will hence be broadened by at best the insignificant amount of $10^{-20} \mathrm{GeV}$ and therefore swerves are irrelevant in the GZK reaction. This type of analysis can be done for many different threshold reactions, for example the scattering of high energy $\mathrm{TeV}$ photons off the infrared background. In all cases the limits on $k$ are strong enough that swerves have no appreciable effect.

\section{CONCLUSION}

LV searches in astrophysics rely not only on assumptions about the nature of the LV model being explored, but also on energy-momentum conservation of the particles involved. In this paper we have argued that causal sets, while intrinsically Lorentz invariant, can technically introduce spurious LV signals if we deal with practical experiments and make the usual assumption that energymomentum is conserved due to the swerve effect. However, we have also shown that existing limits on swerves imply that any errors introduced are negligible at current experimental sensitivity. A bonus is that the diffusion equation (11) that describes the swerve effect is the unique low energy Lorentz invariant diffusion equation. Any statistical process that respects Lorentz invariance but causes fluctuations in energy-momentum should therefore be described by this equation and we can rather generically conclude that any signal of LV in a time of flight or anomalous particle interaction experiment cannot be due instead to a Lorentz invariant modification of translation invariance.

\section{ACKNOWLEDGEMENTS}

We thank Stefano Liberati for useful comments on a draft of this paper.
[1] V. A. Kostelecky and S. Samuel, Phys. Rev. D 39, 683 (1989).

[2] J. R. Ellis, N. E. Mavromatos and D. V. Nanopoulos, arXiv:gr-qc/9909085.

[3] N. E. Mavromatos, arXiv:0708.2250 [hep-th].

[4] R. Gambini and J. Pullin, Phys. Rev. D 59, 124021 (1999) arXiv:gr-qc/9809038.

[5] L. Smolin, Nucl. Phys. B 742, $142 \quad$ (2006) arXiv:hep-th/0501091.

[6] C. Rovelli and S. Speziale, Phys. Rev. D 67, 064019 (2003) arXiv:gr-qc/0205108.

[7] M. Bojowald, H. A. Morales-Tecotl and H. Sahlmann, Phys. Rev. D 71, 084012 (2005) arXiv:gr-qc/0411101.

[8] C. Barcelo, S. Liberati and M. Visser, Living Rev. Rel. 8, 12 (2005) arXiv:gr-qc/0505065.

[9] S. M. Carroll, J. A. Harvey, V. A. Kostelecky, C. D. Lane and T. Okamoto, Phys. Rev. Lett. 87, 141601 (2001)
arXiv:hep-th/0105082.

[10] A. Anisimov, T. Banks, M. Dine and M. Graesser, Phys. Rev. D 65, 085032 (2002) arXiv:hep-ph/0106356.

[11] I. Hinchliffe, N. Kersting and Y. L. Ma, Int. J. Mod. Phys. A 19, 179 (2004) arXiv:hep-ph/0205040.

[12] M. Chaichian, P. Presnajder and A. Tureanu, Phys. Rev. Lett. 94, 151602 (2005) arXiv:hep-th/0409096.

[13] J. Kowalski-Glikman, Lect. Notes Phys. 669, 131 (2005) arXiv:hep-th/0405273.

[14] L. Freidel and J. Kowalski-Glikman, arXiv:0710.2886 [hep-th].

[15] V. A. Kostelecky and C. D. Lane, Phys. Rev. D 60, 116010 (1999) arXiv:hep-ph/9908504.

[16] J. Collins, A. Perez, D. Sudarsky, L. Urrutia and H. Vucetich, Phys. Rev. Lett. 93, 191301 (2004) arXiv:gr-qc/0403053.

[17] P. A. Bolokhov, S. G. Nibbelink and M. Pospelov, Phys. 
Rev. D 72, 015013 (2005) arXiv:hep-ph/0505029.

[18] L. Bombelli, J. Henson and R. D. Sorkin, arXiv:gr-qc/0605006

[19] F. Dowker, J. Henson and R. D. Sorkin, Mod. Phys. Lett. A 19, 1829 (2004) arXiv:gr-qc/0311055.

[20] J. R. Ellis, N. E. Mavromatos and D. V. Nanopoulos, Phys. Rev. D 63, 024024 (2001) arXiv:gr-qc/0007044.

[21] R. Parentani, arXiv:0709.3943 [hep-th].

[22] G. Amelino-Camelia, Y. J. Ng and H. Vanm Dam, Astropart. Phys. 19, 729 (2003) arXiv:gr-qc/0204077.

[23] J. Henson, arXiv:gr-qc/0601121.

[24] R. Aloisio, A. Galante, A. F. Grillo, S. Liberati, E. Luzio and F. Mendez, Phys. Rev. D 74, 085017 (2006) arXiv:gr-qc/0607024.

[25] R. Aloisio, P. Blasi, A. Galante, P. L. Ghia and A. F. Grillo, Astropart. Phys. 19, 127 (2003) arXiv:astro-ph/0205271.

[26] R. D. Sorkin, Annals Phys. 168, 119 (1986).

[27] N. Kaloper and D. Mattingly, Phys. Rev. D 74, 106001
(2006) arXiv:astro-ph/0607485.

[28] D. Colladay and V. A. Kostelecky, Phys. Rev. D 58, 116002 (1998) arXiv:hep-ph/9809521.

[29] R. C. Myers and M. Pospelov, Phys. Rev. Lett. 90, 211601 (2003) arXiv:hep-ph/0301124.

[30] T. Jacobson and D. Mattingly, Phys. Rev. D 64, 024028 (2001) arXiv:gr-qc/0007031.

[31] P. A. Bolokhov and M. Pospelov, arXiv:hep-ph/0703291

[32] D. Mattingly, Living Rev. Rel. 8, 5 (2005) arXiv:gr-qc/0502097.

[33] U. Jacob and T. Piran, Nature Phys. 3, 87 (2007) arXiv:hep-ph/0607145.

[34] M. C. Gonzalez-Garcia and F. Halzen, JCAP 0702, 008 (2007) arXiv:hep-ph/0611359.

[35] R. Abbasi et al. [HiRes Collaboration], arXiv:astro-ph/0703099

[36] T. Yamamoto [Pierre Auger Collaboration], arXiv:0707.2638 [astro-ph]. 